\title{
Treating melanoma in adolescents and young adults: challenges and solutions
}

This article was published in the following Dove Press journal:

Clinical Oncology in Adolescents and Young Adults

15 September 2015

Number of times this article has been viewed

\author{
Radhika Sreeraman Kumar' \\ Jane L Messina ${ }^{2,3}$ \\ Vernon K Sondak ${ }^{3}$ \\ Damon R Reed ${ }^{3-6}$
}

'Department of Radiation Oncology, Moffitt Cancer Center, ${ }^{2}$ Department of Pathology and Cell Biology, Morsani College of Medicine, University of South Florida, ${ }^{3}$ Department of Cutaneous Oncology, ${ }^{4}$ Adolescent and Young Adult Program, ${ }^{5}$ Sarcoma Department, ${ }^{6}$ Chemical Biology and Molecular Medicine Program, Moffitt Cancer Center, Tampa, FL, USA
Correspondence: Damon R Reed Chemical Biology and Molecular Medicine Program, Adolescent and Young Adult Oncology Program, Sarcoma Department, Department of Cutaneous Oncology, Moffitt Cancer Center, I 2902 Magnolia

Drive, Tampa, FL 336 I 2, USA

Tel + I 8I37453242

$\mathrm{Fax}+18137458337$

Email damon.reed@moffitt.org

\begin{abstract}
Systemic therapy of melanoma has made tremendous progress recently for advanced stage patients in terms of excellent, temporary responses through targeting underlying biology of BRAF V600E mutation and affected pathways. A significant subset of patients with advanced disease also has durable responses from immunomodulation through immune checkpoint blockade of CTLA-4 and the PDL-1/PD-1 axis. Clinical trials of these agents included patients as young as 18 years but generally excluded younger adolescents. Ongoing trials in 12-17 year olds have not been accruing as robustly, and as a result available options for these patients are often lagging years behind their adult counterparts. We herein summarize the epidemiology, diagnosis, and multidisciplinary management of adolescents and young adults with melanoma. We offer recommendations to improve the care for adolescent patients with melanoma agespecific evaluations and by including them in up-front melanoma trials.
\end{abstract}

Keywords: melanoma, AYA, systemic therapy, adjuvant therapy

\section{Introduction}

Adolescent and Young Adult (AYA) Oncology encompasses the care of 15- to 39-year-old patients with cancer. These patients have unique needs including fertility preservation, and guidelines have been developed to ensure that a comprehensive approach to these patients can be optimized. ${ }^{1}$ This group has not benefited from clinical trials as much as their younger or older counterparts. ${ }^{2-4}$ Increasingly hospitals are developing programs to address AYA patients' needs. ${ }^{5}$ While literature has supported care for some of these patients according to pediatric protocols, such as in the case of acute lymphoblastic leukemia ${ }^{6}$ and other cancers more commonly treated by pediatric oncologists, ${ }^{7}$ melanoma care for adolescents is likely optimized by these patients being managed in cooperation with comprehensive adult oncology programs experienced with the pathologic evaluation, surgical approaches, and cutting-edge medical therapies that have resulted in enormous gains for melanoma patients in recent years.

There is controversy on the upper and lower age ranges in AYA Oncology and in definition of adolescence in this context. While some argue for a definition of 15-18 years as the lower cut-off point, others argue that after puberty, adolescents as young as 12 years with Tanner Stage 5 development may be sufficiently physiologically mature to enroll in clinical trials. ${ }^{8}$ While the International Conference on Harmonisation guidelines include age $12-16$ or 18 years (with variation among geographic regions), the age range is extended from 12 to 21 years for medical devices. ${ }^{9}$ In fact, a recent study examining drug clearance data since the US Food and Drug Administration (FDA) Amendments Act of 2007 found that more than 94\% of approved agents had 
equivalent dosing in adolescents as in adults when defining adolescence as age 12 years or up. ${ }^{10}$

Melanoma constitutes $7.9 \%$ of all AYA malignancies, making it one of the most common malignancies in this population. ${ }^{11,12}$ Though the incidence of melanoma specific to the AYA population is not readily available, patients younger than 20 comprise $1 \%$ of all melanomas. Incidence steadily rises with age with $6.5 \%$ of all melanoma diagnoses occurring before age 34 years (Figure 1). ${ }^{13}$ The incidence of melanoma in the adolescent population has been increasing at a rate of $2 \%$ per year in the United States over the past 3 decades ${ }^{14}$ with similar increases in European countries. ${ }^{15-17}$

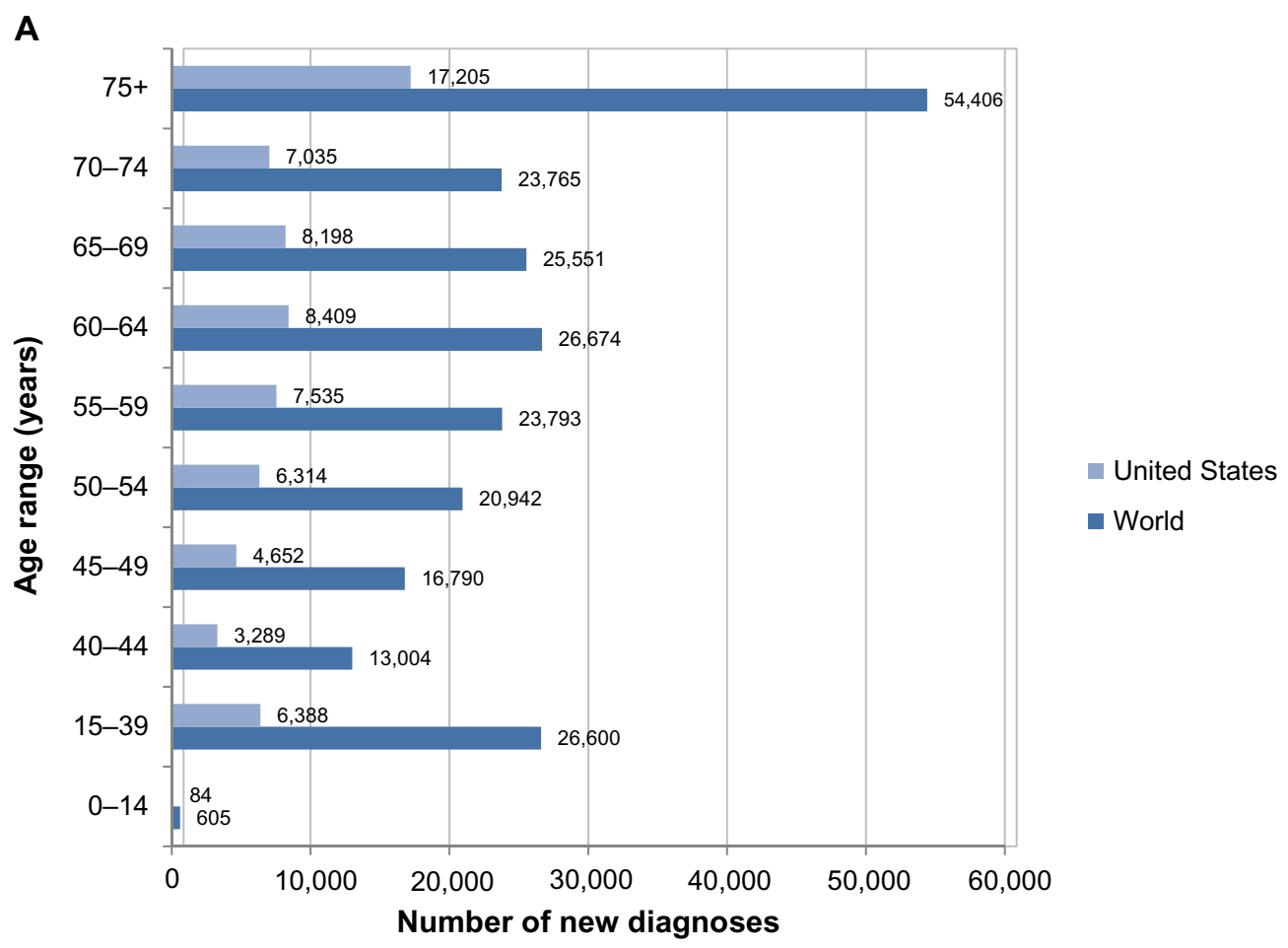

B

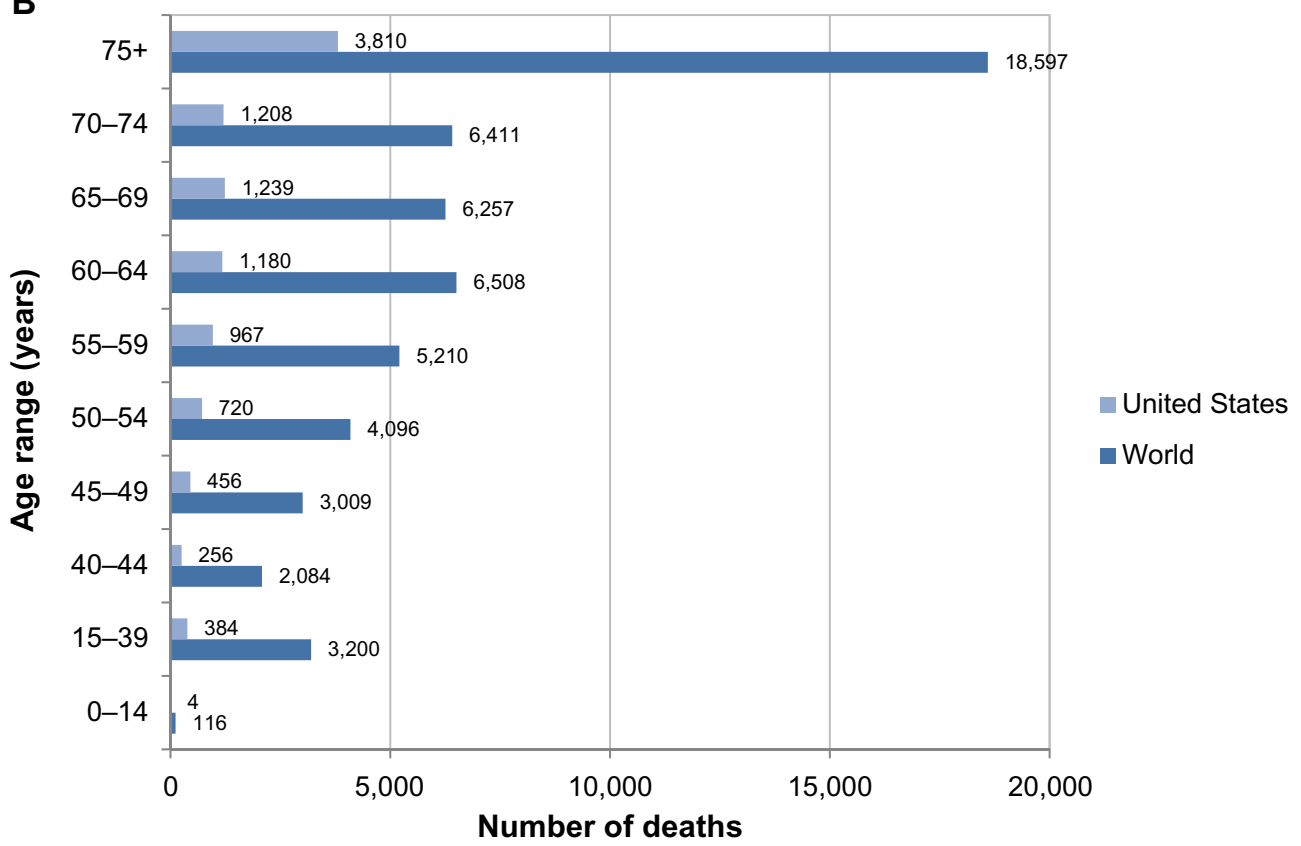

Figure I Incidence and mortality of melanoma.

Notes: Adapted from: GLOBOCAN 2012: Estimated cancer incidence, mortality and prevalence worldwide in 2012. International Agency for Research on Cancer; World Health Organization. Available from: http://globocan.iarc.fr/Pages/age-specific_table_sel.aspx. Accessed August 21,2015 . $^{108}$ (A) Incidence of melanoma by age in the United States and the World in 20I2. Pediatric, adolescent, and young adult patients comprised $9 \%-11 \%$ of all new melanoma diagnoses in 2012 in the United States and in the World. (B) Mortality of melanoma by age in the United States and the World in 2012 . Pediatric, adolescent, and young adult patients comprised $4 \%$ and $6 \%$ of all deaths from melanoma in 2012 both in the United States and in the World. 
While Caucasian patients account for the majority of new diagnoses, the incidence continues to rise in the Hispanic and Native American populations as well. ${ }^{18}$ Risk factors include ultraviolet radiation exposure, tanning bed use, fair skin, family history of melanoma, the presence of multiple atypical nevi, congenital melanocytic nevi, xeroderma pigmentosum, and germline mutations involving cell-cycle mediators. ${ }^{19-21}$

\section{Diagnosis}

\section{Clinical presentation and initial biopsy}

Young adults typically present with classic features of asymmetry, irregular borders, heterogeneous color, and increasing diameter. Their melanomas are more likely to arise from dysplastic nevi that have evolved rather than from congenital nevi. Compared with older adults, they are likely to have localized disease at the time of presentation, more likely to be Caucasian with fair skin, and more likely to have been exposed to tanning beds and UV exposure.

While many adolescents will share the clinical features of adult melanoma, some will have overlapping feature with pyogenic granuloma such as being amelanotic lesions, (pink/red/flesh-colored) with symmetric papular or nodular appearance. Others will be suspicious only because of changes over time, termed evolution. ${ }^{22}$ In fact, the most common prebiopsy diagnosis is pyogenic granuloma. ${ }^{22}$ Due to the non-specific presentation and the relative rarity of AYA melanoma, the potential for delay in diagnosis is high. Adolescent patients are more likely than young adults to have a lesions arise from congenital nevi, be ethnically diverse, and to have germline mutations. Clinical evaluation should include history of a congenital melanocytic nevus or other precursor lesion in the area biopsied, patient's age, extent of biopsy (complete excision, shave biopsy, partial excision/punch), patient's demographics, color and size of the lesion, and a photograph of the lesion, as this can aid the dermatopathologist, further emphasizing the value of a close collaboration between clinician and pathologist in dealing with adolescent pigmented lesions.

Whenever possible, the biopsy specimen should include complete excision of the lesion with a narrow margin of normal skin, which facilitates a pathologic assessment of the lesion that incorporates its relationship to neighboring skin and subcutis.

\section{Pathology}

In young adults, the pathological diagnosis is often unambiguous, and diagnosis can be facilitated with S100, Ki-67, and HMB-45 immunostains. The most common subtypes are superficial spreading and nodular melanomas that arise from dysplastic nevi, and in comparison with older adults, desmoplastic melanoma and lentigo maligna are rare. They are more likely than pediatric melanoma patients to have mutations, such as BRAF, and less likely to have melanomas arising from congenital nevi.

In adolescent patients, there is a broader spectrum of melanocytic neoplasms, sometimes leading to diagnostic ambiguity. While these atypical melanocytic neoplasms occur more frequently in the prepubertal pediatric population, they can be a diagnostic conundrum. Numerous prospective and retrospective studies have sought to elucidate the natural history of these atypical neoplasms ${ }^{23-31}$ further complicated by diagnostic disagreement between even expert dermatopathologists in many cases. ${ }^{32}$

Fluorescent in-situ hybridization can assist the dermatopathologist in determining a diagnosis. In comparison with benign nevi, melanomas in this setting are more likely to have mutations in chromosomes $6 \mathrm{p} 25$ (the locus of gene RREB1), 6q23 (MYB), Cep6 (the centromere of chromosome 6), $11 \mathrm{q} 13$ (CCND1), ${ }^{33}$ 6p25, 11q13, 9p21 (CDKN2A), and 8q24 $(c M Y C) .{ }^{25}$ Melanomas are more likely to have chromosomal gains or losses, with $96 \%$ demonstrating abnormalities. These studies suggest a spectrum of melanocytic neoplasms in the adolescent and pediatric population, ranging from benign nevi to melanoma.

We have found that the lack of a standard nomenclature for classifying these atypical neoplasms leads to long and oftentimes confusing pathology reports, increasing stress among patients and families and potentially making treatment planning more difficult. To improve communication between pathologist and clinician, we have developed a fivepoint scale similar to the Breast Imaging Reporting and Data System classification used for mammography reporting and subsequently modified for describing atypical nevi. ${ }^{34}$ This system (Figure 2) categorizes lesions on a spectrum from 1 (considered unequivocally benign, with no atypical features seen) to 5 (considered unequivocally malignant), based on histopathologic findings and any available molecular/ genetic data. A lesion the pathologist feels is consistent with a benign lesion but has some atypical features is classified as category 2 (atypical, favor benign), while a lesion felt most consistent with malignancy but lacking unequivocal diagnostic features of melanoma is classified as category 4 (atypical, favor melanoma). Atypical lesions without sufficient features to weigh in favor of one or the other end of the spectrum are classified as category 3 (atypical). The specific criteria that make a lesion fall into each category may vary for the different histologic subtypes of atypical melanocytic lesions and even for different pathologists evaluating the same lesion, but the 

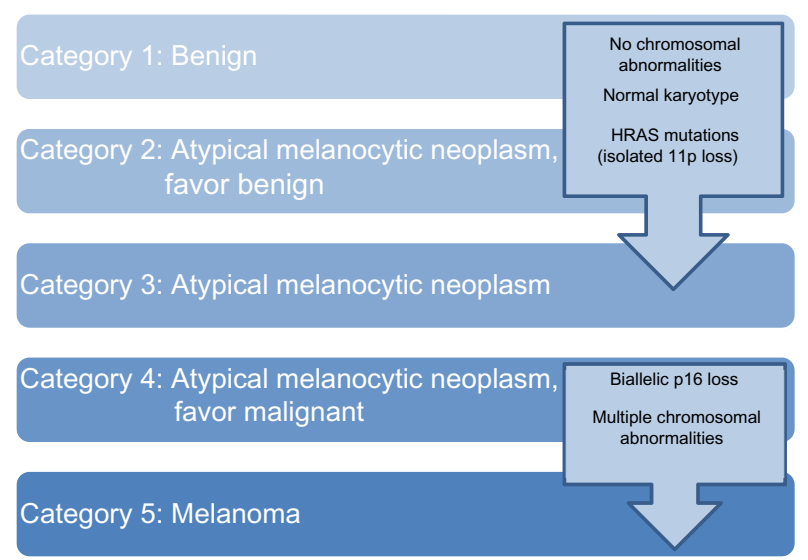

Figure 2 Classification system for pediatric melanocytic neoplasms, from benign to melanoma.

Notes: In addition to histopathologic features, comprehensive genomic hybridization and fluorescent in-situ hybridization can be used to assist in the diagnosis of melanocytic lesions. Benign lesions are less likely to have chromosomal abnormalities, with normal karyotype and those favoring benign more likely to have I Ip loss. The presence of multiple chromosomal abnormalities and biallelic pl 6 loss are more likely to be associated with increasing malignant potential and the diagnosis of unequivocal melanoma.

numbering system allows the clinician to understand exactly how the pathologist classifies that lesion, and plans treatment accordingly. Further information regarding the diagnosis and management of atypical melanocytic neoplasms can be found in several sources. ${ }^{35-38}$ The remainder of the article will be dedicated to the management of AYA patients where an unequivocal diagnosis of melanoma has been made.

\section{Staging}

The American Joint Committee on Cancer staging of melanoma applies across all ages, and there are no specific guidelines for AYA. Due to low risk of distant disease in stage I and II patients without clinical signs or exam findings to suggest spread and due to the risks associated with ionizing radiation in adolescents, ${ }^{39,40}$ computer tomography (CT) or Positron emission tomography/CT scans in patients younger than 18 should be used preoperatively only for well-defined indications: patients with a preoperative diagnosis of stage III melanoma and those with signs or symptoms suspicious of metastatic disease.

\section{Treatment}

\section{Surgery \\ Wide excision}

Surgery is the primary treatment of all localized cutaneous melanoma. There is no optimum excision margin specific to adolescents and young adults with melanoma. Thus, we recommend using the standard adult guidelines with $1 \mathrm{~cm}$ margins for $\leq 1 \mathrm{~mm}$ lesions or where morbidity precludes wider margins in 1-2 $\mathrm{mm}$ depth lesions, and $2 \mathrm{~cm}$ margins for thicker lesions. Younger children may not require as wide a margin even for thicker melanomas. ${ }^{41}$ The primary goal of the excision is to achieve negative margins.

\section{Management of regional lymph nodes}

Younger patients are more likely to have a positive sentinel lymph node biopsy (SLNB), independent of thickness and mitotic activity when compared with older adults. ${ }^{42}$ Over $20 \%$ of pediatric and adolescent patients with clinically negative nodes and a primary melanoma measuring $\geq 1 \mathrm{~mm}$ in thickness will have at least one positive sentinel lymph node. Moreover, SLNB is a prognostic tool for AYA melanoma. Recurrence and death are more likely to occur in sentinel node-positive cases, just as in older adults. ${ }^{43-46}$ The complications of removing a few lymph nodes from a basin are rare in both the AYA and older adult population. ${ }^{47} \mathrm{As}$ such, we routinely advocate the use of SLNB in AYA patients with melanomas thicker than $0.75 \mathrm{~mm}$, just as in adults. ${ }^{48}$

The management of the AYA melanoma patient with a positive sentinel lymph node is similar to that of older adults, but clearly there are some unique considerations to take into account. Limited data are available on the rates of non-sentinel node involvement in pediatric and adolescent melanoma. ${ }^{49,50}$ Although involved non-sentinel lymph nodes are only present in $15 \%-20 \%$ of adult patients, ${ }^{51-53}$ completion lymphadenectomy is the current standard-ofcare recommendation for adult patients. ${ }^{54}$ Complications of a lymph node dissection in AYA patients can include chronic lymphedema and post-operative infections, as in older adults, ${ }^{55}$ so nodal basin observation instead of lymphadenectomy is increasingly being considered. However, adolescents and young adults can face specific issues with compliance with the follow-up recommended for sentinel node-positive patients who do not undergo completion lymphadenectomy.

We advise completion lymphadenectomy or observation selectively based on the following factors: the number and site(s) of sentinel nodes involved with tumor, the extent of tumor involvement within positive nodes, the findings on the preoperative lymphoscintigraphy (which can potentially indicate the likelihood of non-sentinel node involvement), ${ }^{56}$ and based on anticipated ability to be compliant with close follow-up. Patients in our practice with a positive SLNB who choose to be followed without completion lymphadenectomy are recommended to undergo ultrasound surveillance of the node-positive basin every 4-6 months for the first 2-3 years, and then every 6-12 months thereafter for a minimum of 5 years or until development of nodal or distant recurrence. 
All patients with clinically detectable lymph node involvement should undergo radical lymphadenectomy of the involved basin(s) (therapeutic lymph node dissection) unless there is evidence of distant metastatic disease. In the case of inguinal node-positive cases, the indications for pelvic ("deep") node dissection are unclear in adolescents and young adults, as in older adults. Evidence of involvement of one or more iliac or obturator nodes is the sole absolute indication for deep node dissection. However, pelvic lymphadenectomy should be considered for multiple or large inguinal nodes even in the absence of abnormal pelvic nodes on preoperative scanning. Studies in adults have demonstrated that inclusion of the external iliac and obturator nodes with an inguinofemoral node dissection does not increase long-term morbidity, ${ }^{57,58}$ and our institutional experience in the AYA population is comparable.

\section{Systemic treatment}

\section{Metastatic melanoma}

Systemic treatment for unresectable metastatic melanoma for AYA patients is largely extrapolated from studies of older adults. Prior to 2010, no demonstrably effective therapy existed for metastatic or unresectable melanoma. Options for treating patients were limited to dacarbazine and high-dose interleukin-2, ${ }^{59,60}$ neither of which consistently produced improvements in survival. Over the past 5 years, the mutations associated with melanoma have been better characterized, and our understanding of the antitumor immune response has dramatically increased, leading to the development of molecularly targeted therapy and immunotherapy, which have been effective in extending progression-free and overall survival in adults with unresectable metastatic melanoma.

The most important targetable mutation characterized in melanoma is in BRAF, with up to $60 \%$ of melanomas in young adults having point mutations leading to its constitutive activation. ${ }^{61}$ The mutations in adolescent cutaneous melanoma (and to an extent pediatric cases as well) are similar to those of adults. ${ }^{62}$ A mediator of the RAS/RAF/MEK/ ERK pathway, constitutively active BRAF leads to uncontrolled cell proliferation. Targeting BRAF V600 is effective in prolonging progression-free and overall survival ${ }^{63-65}$ The two main agents are vemurafenib and dabrafenib, ${ }^{63,64,66}$ which have been approved by the FDA for unresectable metastatic BRAF-mutated melanoma (Table 1). However, these agents can activate BRAF and the RAS/RAF/MEK/ERK pathway in wild-type BRAF melanomas. As such these agents are reserved for BRAF-mutated melanomas. Resistance can develop with these patients within a median of 6-7 months, ${ }^{63,64}$ and recent trials have attempted to combine agents inhibiting downstream kinases with BRAF inhibitors. Trials combining MEK inhibitors (cobimetinib, trametinib) and BRAF inhibitors (vemurafenib, dabrafenib) have shown promising results in terms of progression-free survival and overall survival $^{67-69}$ (Table 1) and combination therapy is now considered standard-of-care for adult melanoma patients receiving BRAF-targeted therapy. Importantly, virtually all of these trials included only patients 18 years of age and older, excluding adolescent patients.

The other major therapeutic approach has been using immunotherapy. ${ }^{70}$ Immune checkpoint antibodies that reverse the immunosuppressive signals from cytotoxic T-cell antigen-4 (ipilimumab) and PD-1 (nivolumab and pembrolizumab) have been developed. The first of the three to reach clinic was ipilimumab, which has been shown to improve overall and progression-free survival in patients with metastatic melanoma in comparison with a vaccine or dacarbazine. ${ }^{71,72}$ When the results of Phases II and III trials of ipilimumab were pooled and analyzed, there was median overall survival of 11.4 months, and at 3 years, $22 \%$ of all metastatic melanoma patients were still alive. ${ }^{73,74}$ Nivolumab and pembrolizumab are antibodies targeting PD-1 on cytotoxic T-lymphocytes. Nivolumab has been shown to improve progression-free survival both as a single agent in comparison with dacarbazine and combined with ipilimumab compared with ipilimumab alone in patients with unresectable metastatic melanoma. ${ }^{75-77}$ Similarly, pembrolizumab demonstrated a significant improvement in progression-free and overall survival in comparison with ipilimumab (Table 1). ${ }^{78}$

These agents are being investigated in the pediatric population as well, but typically after adult studies have been completed. Ipilumimab results in adults were first reported in Phase I studies in 2005, and the pivotal Phase III study accrued 676 patients over a 4-year period, a rate of more than 14 patients per month. ${ }^{71}$ The pediatric Phase I ipilimumab study only began accrual in September of 2011 and accrued just 29 patients through completion in December 2014 (NCT01445379). Thus, there has been a 7- to 10-year gap between adult and adolescent exposure to ipilimumab for melanoma with a continuing gap in ongoing combination studies, which do not include adolescent patients. As outcomes and toxicities are not reported by age, results and toxicities specific to AYA patients such as fertility and quality of life are undefined. Furthermore subgroup analyses, even post hoc, for the recently completed trials would be 


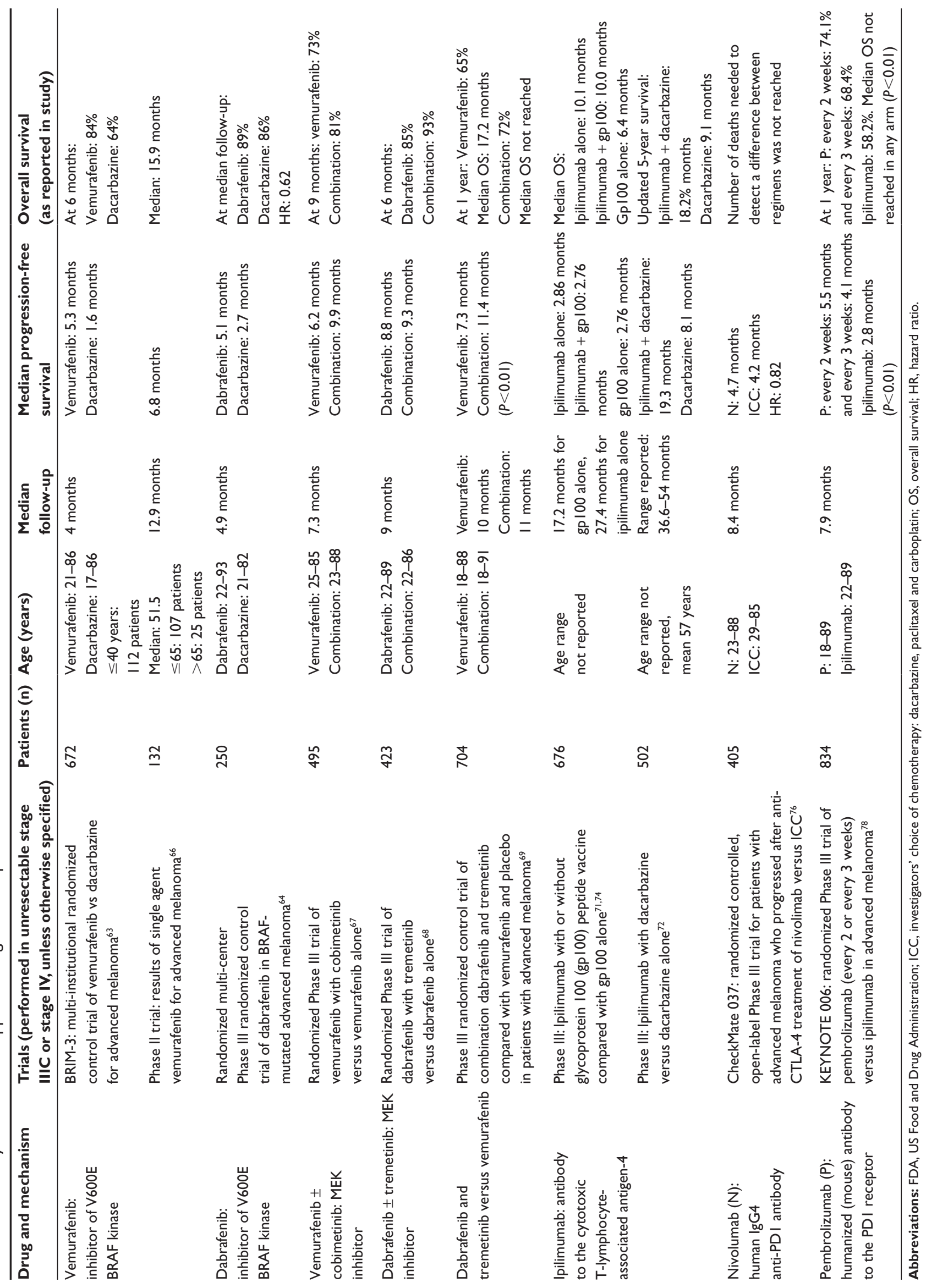


worthwhile to see whether age independently affects toxicity (traditionally reported, but also fertility) or outcome of checkpoint inhibitors.

\section{Adjuvant therapy}

Systemic adjuvant therapy has been used in the older adult melanoma population for stage III and selected high-risk stage II patients. In adults, targeted agents are now being explored in the adjuvant setting. Ipilimumab has been studied as adjuvant systemic treatment. EORTC 18071, a Phase III trial, investigated adjuvant ipilimumab for 3 years versus placebo after complete resection and lymphadenectomy of highrisk stage III cutaneous melanoma in adults. The trial included patients over the age of 18 years. Patients receiving ipilimumab had a significantly improved median recurrence-free survival of 26.1 months in comparison with 17.1 months for those receiving placebo. When stratified by tumor characteristics, patients with microscopic lymph node involvement and ulcerated primaries appeared to have more benefit. However, substantial increased adverse events were reported in the ipilimumab arm, with more than $50 \%$ of patients developing grade 3 or grade 4 toxicities. Gastrointestinal, hepatic, and endocrine toxicities resulted in discontinuation of treatment in over $50 \%$ of the patients in the ipilimumab arm, mostly within the first 3 months. Five deaths in the ipilimumab arm were related to adverse drug events: three were from colitis, one from myocarditis, and one from multi-organ failure associated with Guillain-Barre Syndrome. ${ }^{79}$ However, the age of these patients was not reported.

Like treatment for metastatic disease, adjuvant trials for adolescents have often lagged behind adult trials. While recent cooperative group Phase III studies (SWOG trial S0008 [NCT00006237], ECOG E1697 [NCT00003641], and E1609 [NCT012734338]) investigating the role of adjuvant interferon in patients with node positive melanoma have included children under 18 years of age, no results specific for adolescent patients have been published. The same cooperative groups should be applauded for ECOG 1609, an ongoing adjuvant trial for high-risk stage III/IV melanoma randomizing patients to high-dose interferon versus ipilimumab at $10 \mathrm{mg} / \mathrm{kg}$ or $3 \mathrm{mg} / \mathrm{kg}$, which is enrolling adolescents from age 15 years (NCT01274338). The most studied agent in the AYA population is high-dose interferon $\alpha-2 b$, but data specific to the AYA population are lacking. Pediatric reports are small and suggest better tolerance of this agent in younger populations. ${ }^{80-82}$ Adult studies have been reported but conclusions regarding the AYA melanoma population cannot be drawn as age-specific outcomes or toxicities are not reported. ${ }^{83}$

\section{Radiation therapy \\ Metastatic melanoma}

For metastatic melanoma in adults, radiation has a particular value in local control and in oligometastatic disease either alone or with systemic treatment. Stereotactic radiosurgery has been noted in multiple prospective and retrospective studies to have local control rates up to $90 \%$ at the treatment sites with a favorable safety profile, particularly in brain, ${ }^{84}$ spine, ${ }^{85}$ and lung. Moreover, both clinically and in animal studies, radiation delivered with a high dose per fraction appears to have an abscopal effect, with radiation inducing an immune response. ${ }^{86-88}$ The mechanism has not yet been completely understood. ${ }^{89}$ Studies have explored and are ongoing to elucidate a possible synergistic effect between stereotactic radiation and new immunotherapeutic agents both preclinical ${ }^{90}$ and clinical (NCT01449279, NCT01689974, NCT01557114, NCT01565837, and NCT01497808). Similarly, the potential toxicities of the interaction between immunotherapy and stereotactic radiation are yet to be determined when the two modalities are used concurrently.

\section{Adjuvant radiation}

The role of adjuvant radiation therapy in adult melanoma is controversial. Retrospective as well as prospective trials have demonstrated regional control rates greater than $80 \%$ at 5 years. ${ }^{91-95}$ There has been one single randomized control trial comparing adjuvant radiation versus observation after completion lymphadenectomy in patients with high-risk node positive disease. ${ }^{96}$ Despite significant improvement in local control and reduction in recurrence by more than $50 \%$, there was no benefit in overall survival with radiation, as the majority of recurrences were distant or out of the treatment field. When axillary recurrences were analyzed, patients receiving radiation were more likely to have adjacent field recurrence while those who were observed were more likely to have an infield recurrence..$^{97}$

As with other therapies, there is little information available specifically in the AYA population. In the patients younger than 18, in the absence of an overall survival benefit, radiation therapy is often deferred until recurrence or in the case of metastasis.

Advances in radiation techniques such as image guidance, stereotactic radiation therapy, intensity modulated radiation, and proton beam radiation have allowed for more targeted, conformal treatment. The sparing of normal tissue can have a particular benefit in younger patients. Retrospective studies suggest equivalence of hypofractionated and conventional treatment fractionation. ${ }^{95,98}$ However, side effects of adjuvant 
radiation therapy can include the risk of lymphedema, particularly with axillary and inguinal radiation where up to $50 \%$ of patients can experience lymphedema. In a recent study of 20 patients receiving adjuvant radiation to the axilla and inguinal regions, 15 of whom received intensity modulated radiation therapy, the rate of Grade 1-2 lymphedema was $45 \%$ with one incident of Grade 3 lymphedema. Of the $65 \%$ of patients treated who had a recurrence, the majority were distant metastases. ${ }^{99}$ Thus, adjuvant radiation in adults is reserved for high-risk patients: multiple nodes (greater than 3 for the axilla and greater than 4 for the inguinal region), extracapsular extension, lesions where resection to negative margins would result in unacceptable morbidity and recurrent disease. In patients younger than 18 years of age, the threshold for adjuvant radiation is even higher, with residual disease or unresectable disease being the main indication in non-metastatic patients.

\section{AYA-specific management Fertility preservation}

Additional consideration in treatment in the AYA setting involves the discussion of fertility preservation. Chemotherapy and radiation to the pelvis without shielding can affect fertility. The effects of immunotherapy or targeted therapies on fertility have not been well characterized. Dabrafenib, imatinib, and ipilimumab are thought to affect pregnancy by crossing the placental membrane like maternal antibodies, while the effects of vemurafenib on fertility are yet to be determined. ${ }^{100}$ Often these patients do not know that their fertility may be at risk, did not have access to information regarding possible options, did not have adequate financial resources, or did not have adequate time to undergo treatment. ${ }^{101}$ For female AYA patients, embryo preservation and cryopreservation of oocytes or ovarian tissue can be considered prior to systemic treatment and ovarian transposition and gonadal shielding can be considered prior to pelvic radiation. For male AYA patients, sperm banking and gonadal shielding during pelvic radiation can be considered. While successful treatment should be the primary focus, fertility should be discussed prior to the initiation of treatment.

\section{Survivorship}

There are no specific guidelines for follow-up in AYA melanoma, and the guidelines set by the National Comprehensive Cancer Network for melanoma are used. ${ }^{1,102}$ The possibility of recurrence more than 5 years after diagnosis reflecting the long natural history of melanoma in adolescents points to the need for long-term follow-up, which can be particularly challenging in the AYA population. ${ }^{36}$ Patients with earlystage localized disease have an excellent prognosis with more than $90 \%$ overall survival in stage I disease and 79\%-100\% for stage II disease..$^{21,46,103}$ These patients should undergo an annual skin examination with a dermatologist, in most cases as well as follow-up every 3-12 months for a minimum of 5 years and annual follow-up thereafter, given the potential for late recurrence. In adolescents as in older adults, routine imaging is not required. For patients with regional metastatic disease (stage III), overall survival at 10 years is $70 \%-77 \%$. ${ }^{46}$ Closer follow-up is recommended with history and physical to be performed every 3-6 months for the first 2 years, every 3-12 months until 5 years and annually thereafter. Imaging can be considered in these patients. Stage IV disease portends a dismal overall survival. In the rare metastatic patients resected to a disease-free state, follow-up similar to that described for stage III patients is warranted.

\section{Socio-economic challenges}

AYA patients also face economic and social barriers. While adolescents are often insured through their parents, young adults are historically more likely to be underinsured or uninsured despite the recent Affordable Care Act. ${ }^{2}$ When adjusted for age, sex, zip code, level of education, race and facility type, young adult Medicaid patients with skin cancers are six times more likely to present with distant disease, and uninsured patients are 3.5 times more likely to present with distant metastases as compared to patients with private insurance. ${ }^{11}$ In general, AYA patients are less likely to be treated at a National Cancer Institute designated cancer center and more likely to experience gaps in access to care within the first 3 years of diagnosis. Risk factors for gaps in coverage include lower educational level, currently receiving definitive treatment and poor health prior to diagnosis. Even $20 \%$ of those with insurance report that they have had to forgo some physician recommended treatments due to undercoverage or lack of financial resources. ${ }^{104}$ Importantly, these patients are also less likely to enroll in clinical studies, ${ }^{105}$ one of the key reasons thought to contribute to the lack of improvements in survival in this population.

While adolescents are frequently excluded from clinical trials due to age, young adults have to contend with multiple competing obligations such as supporting a family, education, and holding stable employment. ${ }^{106}$ A survey of AYA patients and their family members revealed that increased treatment time, number of procedures, and amount of information given were potential barriers to enrollment. The stress of diagnosis also led adolescents and young adult to defer to 
their parents and family members with regard to clinical decision making. ${ }^{107} \mathrm{~A}$ conscious effort with both the patients and their families must be made to promote clinical trial enrollment by AYA melanoma patients.

\section{Future directions}

Treatment of AYA melanoma will likely continue to be based on extrapolation from data in trials heavily dependent on the enrollment of older adults. Based on the physiology of AYA patients and our current understanding of the biology of melanoma in the populations, we would expect the adolescent population to achieve similar benefits from the newer therapies as those published in Table 1. Trials lagging nearly 1 decade behind adult studies have not allowed adolescents to experience the most current potential benefits, specifically the current generation of combination clinical trials in this quickly adapting field with a recent remarkable track record of successes. Furthermore, if standards of care continue to change at the current rate and adult trials continue to accrues patients at orders of magnitude faster than pediatric and adolescent trials, then single agent studies at pediatric centers may not be feasible at their onset, as physicians and families may select off-label use of newer combinations over pediatric clinical trials based on published data. In addition to excluding adolescent patients from cutting edge trials, few outcomes such as fertility and drug toxicities or quality of life changes specific to the AYA population have thus far been reported. ${ }^{100}$

Thus the solutions that we propose for AYA patients, especially adolescents include increasing access to future clinical trials by reducing the lower age limits for inclusion. This would eliminate the need to repeat the same trials later at pediatric centers while concurrent adult trials innovate by combining therapies with potentially greater survival benefit. Second, including age-specific analyses regarding fertility effects and quality of life measures would be beneficial for this subset of patients. Third, we would also recommend post hoc analyses of the plethora of completed recent trials by age in order to evaluate disease outcome, side effect profile, and adherence by age. Fourth, we strongly advocate for greater enrollment of young adults on the current unresectable and adjuvant melanoma trials through patient and physician education on this unique patient population. The AYA patient population has historically not participated in clinical trials as much as older adults. In the recent history of melanoma therapy this means that significant opportunities for durable remission may have been missed. The best way to improve adolescent melanoma care may well be to improve their access to the latest clinical trials and thus be able to provide access to these agents when FDA approved.

\section{Disclosure}

VKS is a consultant for Amgen, Bristol Myers-Squibb, GlaxoSmithKline, Merck, Navidea, Novartis, and Provectus. JLM is a consultant for GlaxoSmithKline and Durect Corporation. The authors report no other conflicts of interest in this work.

\section{References}

1. Coccia PF, Pappo AS, Altman J, et al. Adolescent and young adult oncology, version 2.2014. J Natl Compr Canc Netw. 2014;12(1):21-32; quiz 32.

2. Bleyer A, Ulrich C, Martin S. Young adults, cancer, health insurance, socioeconomic status, and the Patient Protection and Affordable Care Act. Cancer. 2012;118(24):6018-6021.

3. Bleyer A, Montello M, Budd T, Saxman S. National survival trends of young adults with sarcoma - lack of progress is associated with lack of clinical trial participation. Cancer. 2005;103(9):1891-1897.

4. Bleyer A, Budd T, Montello M. Adolescents and young adults with cancer: the scope of the problem and criticality of clinical trials. Cancer. 2006;107(7 Suppl):1645-1655.

5. Reed D, Block RG, Johnson R. Creating an adolescent and young adult cancer program: lessons learned from pediatric and adult oncology practice bases. J Natl Compr Canc Netw. 2014;12(10): 1409-1415.

6. Rizzari C, Putti MC, Colombini A, et al. Rationale for a pediatricinspired approach in the adolescent and young adult population with acute lymphoblastic leukemia, with a focus on asparaginase treatment. Hematol Rep. 2014;6(3):5554.

7. Bleyer A. The Quid Pro Quo of pediatric versus adult services for older adolescent cancer patients. Pediatr Blood Cancer. 2010;54(2): 238-241.

8. ICH. Guidance for Industry E11 Clinical Investigation of Medicinal Products in the Pediatric Population. 2000. Available from: http:// www.fda.gov/downloads/Drugs/GuidanceComplianceRegulatoryInformation/Guidances/UCM073143.pdf. Accessed May 29, 2015.

9. Field MJ, Behrman RE, Committee on Clinical Research Involving Children. The necessity and challenges of clinical research involving children. In: Institute of Medicine (US) Committee on Clinical Research Involving Children, Field MJ, Behrman RE, editors. Ethical Conduct of Clinical Research Involving Children. Washington, DC, USA: National Academies Press; 2004:64-66.

10. Momper JD, Mulugeta Y, Green DJ, et al. Adolescent dosing and labeling since the Food and Drug Administration Amendments Act of 2007. JAMA Pediatr. 2013;167(10):926-932.

11. Robbins AS, Lerro CC, Barr RD. Insurance status and distant-stage disease at diagnosis among adolescent and young adult patients with cancer aged 15 to 39 years: National Cancer Data Base, 2004 through 2010. Cancer. 2014;120(8):1212-1219.

12. American Cancer Society. Melanoma Skin Cancer. 2015. Available from: http://www.cancer.org/cancer/skincancer-melanoma/detailedguide/ melanoma-skin-cancer-key-statistics. Accessed June 4, 2015.

13. National Cancer Institute. Surveillance, Epidemiology, and End Results Program: SEER Stat Fact Sheets: Melanoma of the Skin [webpage on the Internet]. 2015. Available from: http://seer.cancer.gov/statfacts/html/ melan.html. Accessed June 1, 2015.

14. Austin MT, Xing Y, Hayes-Jordan AA, Lally KP, Cormier JN. Melanoma incidence rises for children and adolescents: an epidemiologic review of pediatric melanoma in the United States. J Pediatr Surg. 2013;48(11):2207-2213. 
15. Helvind NM, Holmich LR, Smith S, et al. Incidence of in situ and invasive melanoma in Denmark from 1985 through 2012: a National Database Study of 24059 melanoma cases. JAMA Dermatol. Epub 2015 Jun 10.

16. AIRTUM Working Group, CCM, AIEOP Working Group. Italian cancer figures, report 2012: cancer in children and adolescents. Epidemiol Prev. 2013;37(1 Suppl 1):1-225.

17. Erdmann F, Lortet-Tieulent J, Schuz J, et al. International trends in the incidence of malignant melanoma 1953-2008 - are recent generations at higher or lower risk? Int J Cancer. 2013;132(2):385-400.

18. Rajput A, Faizi SA, Nir I, et al. Pediatric melanoma in New Mexico American Indians, Hispanics, and non-Hispanic whites, 1981-2009. Am J Surg. 2014;207(3):412-416; discussion 416.

19. Ferrari A, Bono A, Baldi M, et al. Does melanoma behave differently in younger children than in adults? A retrospective study of 33 cases of childhood melanoma from a single institution. Pediatrics. 2005;115(3):649-654.

20. Pappo AS. Melanoma in children and adolescents. Eur J Cancer. 2003;39(18):2651-2661.

21. Lange JR PB, Chang DC, Soong SJ, Balch CM. Melanoma in children and teenagers: an analysis of patients from the National Cancer Database. J Clin Oncol. 2007;25(11):1363-1368.

22. Cordoro KM, Gupta D, Frieden IJ, McCalmont T, Kashani-Sabet M. Pediatric melanoma: results of a large cohort study and proposal for modified ABCD detection criteria for children. J Am Acad Dermatol. 2013;68(6):913-925.

23. Cerrato F, Wallins JS, Webb ML, McCarty ER, Schmidt BA, Labow BI. Outcomes in pediatric atypical spitz tumors treated without sentinel lymph node biopsy. Pediatr Dermatol. 2012;29(4):448-453.

24. DeDavid M, Orlow SJ, Provost N, et al. A study of large congenital melanocytic nevi and associated malignant melanomas: review of cases in the New York University Registry and the world literature. J Am Acad Dermatol. 1997;36(3 Pt 1):409-416.

25. Gerami P, Cooper C, Bajaj S, et al. Outcomes of atypical spitz tumors with chromosomal copy number aberrations and conventional melanomas in children. Am J Surg Pathol. 2013;37(9): 1387-1394.

26. McCormack CJ, Conyers RK, Scolyer RA, et al. Atypical Spitzoid neoplasms: a review of potential markers of biological behavior including sentinel node biopsy. Melanoma Res. 2014;24(5):437-447.

27. Moscarella E, Zalaudek I, Cerroni L, et al. Excised melanocytic lesions in children and adolescents - a 10-year survey. Br J Dermatol. 2012;167(2):368-373.

28. Barnhill RL. The Spitzoid lesion: rethinking Spitz tumors, atypical variants, 'Spitzoid melanoma' and risk assessment. Mod Pathol. 2006;19(Suppl 2):S21-S33.

29. Spatz A, Calonje E, Handfield-Jones S, Barnhill RL. Spitz tumors in children: a grading system for risk stratification. Arch Dermatol. 1999;135(3):282-285.

30. Tannous ZS, Mihm MC Jr, Sober AJ, Duncan LM. Congenital melanocytic nevi: clinical and histopathologic features, risk of melanoma, and clinical management. J Am Acad Dermatol. 2005;52(2): 197-203.

31. Zaal LH, Mooi WJ, Klip H, van der Horst CM. Risk of malignant transformation of congenital melanocytic nevi: a retrospective nationwide study from The Netherlands. Plast Reconstr Surg. 2005; 116(7):1902-1909.

32. Gerami P, Busam K, Cochran A, et al. Histomorphologic assessment and interobserver diagnostic reproducibility of atypical spitzoid melanocytic neoplasms with long-term follow-up. Am J Surg Pathol. 2014;38(7):934-940.

33. Gerami P, Wass A, Mafee M, Fang Y, Pulitzer MP, Busam KJ. Fluorescence in situ hybridization for distinguishing nevoid melanomas from mitotically active nevi. Am J Surg Pathol. 2009;33(12): $1783-1788$

34. Piepkorn MW, Barnhill RL, Elder DE, et al. The MPATH-Dx reporting schema for melanocytic proliferations and melanoma. J Am Acad Dermatol. 2014;70(1):131-141.
35. Reed D, Kudchadkar R, Zager JS, Sondak VK, Messina JL. Controversies in the evaluation and management of atypical melanocytic proliferations in children, adolescents, and young adults. J Natl Compr Canc Netw. 2013;11(6):679-686.

36. Mills OL, Marzban S, Zager JS, Sondak VK, Messina JL. Sentinel node biopsy in atypical melanocytic neoplasms in childhood: a single institution experience in 24 patients. J Cutan Pathol. 2012;39(3): 331-336.

37. Sreeraman Kumar RM JL, Reed D, Navid F, Sondak VK. Pediatric melanoma and atypical melanocytic neoplasms. In: Kaufman HL, editor. Cancer Treatment and Research: Melanoma. New York (NY): Springer; 2015.

38. Sondak VK, Reed DR, Messina JL. A comprehensive approach to pediatric atypical melanocytic neoplasms, with comment on the role of sentinel lymph node biopsy. Crit Rev Oncog. In press 2015.

39. Miglioretti DL, Johnson E, Williams A, et al. The use of computed tomography in pediatrics and the associated radiation exposure and estimated cancer risk. JAMA Pediatr. 2013;167(8):700-707.

40. Pearce MS, Salotti JA, Little MP, et al. Radiation exposure from CT scans in childhood and subsequent risk of leukaemia and brain tumours: a retrospective cohort study. Lancet. 2012;380(9840):499-505.

41. Wong JY, Sondak VK. Unanswered questions about margin recommendations for primary cutaneous melanoma. J Natl Compr Canc Netw. 2012;10(3):357-365.

42. Sondak VK, Taylor JM, Sabel MS, et al. Mitotic rate and younger age are predictors of sentinel lymph node positivity: lessons learned from the generation of a probabilistic model. Ann Surg Oncol. 2004;11(3): 247-258.

43. Morton DL, Thompson JF, Cochran AJ, et al. Final trial report of sentinel-node biopsy versus nodal observation in melanoma. $N$ Engl $J$ Med. 2014;370(7):599-609.

44. Han D, Zager JS, Han G, et al. The unique clinical characteristics of melanoma diagnosed in children. Ann Surg Oncol. 2012;19(12): 3888-3895.

45. Han D, Turner LM, Reed D, Messina JL, Sondak VK. The prognostic significance of lymph node metastasis in pediatric melanoma and atypical melanocytic proliferations. Expert Rev Dermatol. 2013;8(2):103-106.

46. Averbook BJ, Lee SJ, Delman KA, et al. Pediatric melanoma: analysis of an international registry. Cancer. 2013;119(22):4012-4019.

47. Palmer PE 3rd, Warneke CL, Hayes-Jordan AA, et al. Complications in the surgical treatment of pediatric melanoma. J Pediatr Surg. 2013;48(6):1249-1253.

48. Han D, Zager JS, Shyr Y, et al. Clinicopathologic predictors of sentinel lymph node metastasis in thin melanoma. J Clin Oncol. 2013;31(35):4387-4393.

49. Howman-Giles R, Shaw HM, Scolyer RA, et al. Sentinel lymph node biopsy in pediatric and adolescent cutaneous melanoma patients. Ann Surg Oncol. 2010;17(1):138-143.

50. Urso C, Borgognoni L, Doria M, Tinacci G, Zini E. Non-sentinel lymph node involvement in a patient with an atypical Spitz tumor and a positive sentinel node. Report of a case and review of the literature. J Cutan Pathol. 2009;36(5):586-590.

51. Rossi CR, De Salvo GL, Bonandini E, et al. Factors predictive of nonsentinel lymph node involvement and clinical outcome in melanoma patients with metastatic sentinel lymph node. Ann Surg Oncol. 2008;15(4):1202-1210.

52. McMasters KM, Wong SL, Edwards MJ, et al. Frequency of nonsentinel lymph node metastasis in melanoma. Ann Surg Oncol. 2002;9(2): 137-141.

53. Murali R, Desilva C, Thompson JF, Scolyer RA. Non-Sentinel Node Risk Score (N-SNORE): a scoring system for accurately stratifying risk of non-sentinel node positivity in patients with cutaneous melanoma with positive sentinel lymph nodes. J Clin Oncol. 2010;28(29):4441-4449.

54. Wong SL, Balch CM, Hurley P, et al. Sentinel lymph node biopsy for melanoma: American Society of Clinical Oncology and Society of Surgical Oncology joint clinical practice guideline. J Clin Oncol. 2012; 30(23):2912-2918. 
55. Roaten JB, Partrick DA, Pearlman N, Gonzalez RJ, Gonzalez R, McCarter MD. Sentinel lymph node biopsy for melanoma and other melanocytic tumors in adolescents. J Pediatr Surg. 2005;40(1):232-235.

56. van der Ploeg IM, Valdes Olmos RA, Kroon BB, Nieweg OE. Tumorpositive sentinel node biopsy of the groin in clinically node-negative melanoma patients: superficial or superficial and deep lymph node dissection? Ann Surg Oncol. 2008;15(5):1485-1491.

57. Faries MB, Thompson JF, Cochran A, et al. The impact on morbidity and length of stay of early versus delayed complete lymphadenectomy in melanoma: results of the Multicenter Selective Lymphadenectomy Trial (I). Ann Surg Oncol. 2010;17(12):3324-3329.

58. Sarnaik AA, Puleo CA, Zager JS, Sondak VK. Limiting the morbidity of inguinal lymphadenectomy for metastatic melanoma. Cancer Control. 2009; 16(3):240-247.

59. Sondak VK, Gibney GT. Indications and options for systemic therapy in melanoma. Surg Clin North Am. 2014;94(5):1049-1058.

60. Demierre MF, Sabel MS, Margolin KA, Daud AI, Sondak VK. State of the science 60 th anniversary review: 60 Years of advances in cutaneous melanoma epidemiology, diagnosis and treatment, as reported in the journal Cancer. Cancer. 2008;1;113(7 Suppl):1728-1743.

61. Lee JH, Choi JW, Kim YS. Frequencies of BRAF and NRAS mutations are different in histological types and sites of origin of cutaneous melanoma: a meta-analysis. Br J Dermatol. 2011;164(4):776-784.

62. Lu C, Zhang J, Nagahawatte P, et al. The genomic landscape of childhood and adolescent melanoma. J Invest Dermatol. 2015;135(3):816-823.

63. Chapman PB, Hauschild A, Robert C, et al. Improved survival with vemurafenib in melanoma with BRAF V600E mutation. $N$ Engl J Med. 2011;364(26):2507-2516.

64. Hauschild A, Grob JJ, Demidov LV, et al. Dabrafenib in BRAF-mutated metastatic melanoma: a multicentre, open-label, phase 3 randomised controlled trial. Lancet. 2012;380(9839):358-365.

65. Flaherty KT, Puzanov I, Kim KB, et al. Inhibition of mutated, activated BRAF in metastatic melanoma. $N$ Engl J Med. 2010;363(9):809-819.

66. Sosman JA, Kim KB, Schuchter L, et al. Survival in BRAF V600-mutant advanced melanoma treated with vemurafenib. $N$ Engl J Med. 2012; 366(8):707-714.

67. Larkin J, Ascierto PA, Dreno B, et al. Combined vemurafenib and cobimetinib in BRAF-mutated melanoma. N Engl J Med. 2014;371(20): 1867-1876.

68. Long GV, Stroyakovskiy D, Gogas H, et al. Combined BRAF and MEK inhibition versus BRAF inhibition alone in melanoma. $N$ Engl J Med. 2014;371(20):1877-1888.

69. Robert C, Karaszewska B, Schachter J, et al. Improved overall survival in melanoma with combined dabrafenib and trametinib. NEngl J Med. 2015;372(1):30-39.

70. McArthur GA, Ribas A. Targeting oncogenic drivers and the immune system in melanoma. J Clin Oncol. 2013;31(4):499-506.

71. Hodi FS, O'Day SJ, McDermott DF, et al. Improved survival with ipilimumab in patients with metastatic melanoma. $N$ Engl J Med. 2010;363(8):711-723.

72. Robert C, Thomas L, Bondarenko I, et al. Ipilimumab plus dacarbazine for previously untreated metastatic melanoma. $N$ Engl J Med. 2011;364(26):2517-2526

73. Schadendorf D, Hodi FS, Robert C, et al. Pooled analysis of long-term survival data from phase II and phase III trials of ipilimumab in unresectable or metastatic melanoma. J Clin Oncol. 2015;33(17):1889-1894.

74. Maio M, Grob JJ, Aamdal S, et al. Five-year survival rates for treatmentnaive patients with advanced melanoma who received ipilimumab plus dacarbazine in a phase III trial. J Clin Oncol. 2015;33(10): 1191-1196.

75. Robert C, Long GV, Brady B, et al. Nivolimab in previously untreated melanoma without BRAF mutation. New Engl J Med. 2015;372(4): 320-330.

76. Weber JS, D'Angelo SP, Minor D, et al. Nivolumab versus chemotherapy in patients with advanced melanoma who progressed after anti-CTLA-4 treatment (CheckMate 037): a randomised, controlled, open-label, phase 3 trial. Lancet Oncol. 2015;16(4):375-384.
77. Postow MA, Chesney J, Pavlick AC, et al. Nivolumab and ipilimumab versus ipilimumab in untreated melanoma. $N$ Engl J Med. 2015; 372(21):2006-2017.

78. Robert C, Schachter J, Long GV, et al. Pembrolizumab versus ipilimumab in advanced melanoma. $N$ Engl $J$ Med. 2015;372(26): 2521-2532.

79. Eggermont AMM, Chiarion-Sileni V, Grob JJ, et al. Adjuvant ipilimumab versus placebo after complete resection of high-risk stage III melanoma (EORTC 18071): a randomised, double-blind, phase 3 trial. Lancet Oncol. 2015;16(5):522-530.

80. Shah NC, Gerstle JT, Stuart M, Winter C, Pappo A. Use of sentinel lymph node biopsy and high-dose interferon in pediatric patients with high-risk melanoma: the Hospital for Sick Children experience. $J$ Pediatr Hematol Oncol. 2006;28(8):496-500.

81. Chao MM, Schwartz JL, Wechsler DS, Thornburg CD, Griffith KA, Williams JA. High-risk surgically resected pediatric melanoma and adjuvant interferon therapy. Pediatr Blood Cancer. 2005;44(5): 441-448.

82. Navid F, Furman WL, Fleming M, et al. The feasibility of adjuvant interferon alpha-2b in children with high-risk melanoma. Cancer. 2005;103(4):780-787.

83. Kirkwood JM, Strawderman MH, Ernstoff MS, Smith TJ, Borden EC, Blum RH. Interferon alfa-2b adjuvant therapy of high-risk resected cutaneous melanoma: the Eastern Cooperative Oncology Group Trial EST 1684. J Clin Oncol. 1996;14(1):7-17.

84. Mathieu D, Kondziolka D, Cooper PB, et al. Gamma knife radiosurgery in the management of malignant melanoma brain metastases. Neurosurgery. 2007;60(3):471-481; discussion 481-472.

85. Gerszten PC, Burton SA, Quinn AE, Agarwala SS, Kirkwood JM Radiosurgery for the treatment of spinal melanoma metastases. Stereotact Funct Neurosurg. 2005;83(5-6):213-221.

86. Demaria $\mathrm{S}, \mathrm{Ng} \mathrm{B}$, Devitt $\mathrm{ML}$, et al. Ionizing radiation inhibition of distant untreated tumors (abscopal effect) is immune mediated. Int $J$ Radiat Oncol Biol Phys. 2004;58(3):862-870.

87. Postow MA, Callahan MK, Barker CA, et al. Immunologic correlates of the abscopal effect in a patient with melanoma. $N$ Engl J Med. 2012;366(10):925-931.

88. Park SS, Dong H, Zhao W, et al. PD-1 blockade enhances radiation therapy-induced abscopal effect. Int J Radiat Oncol Biol Phys. 2014; 90(1):S57-S58.

89. Twyman-Saint Victor C, Rech AJ, Maity A, et al. Radiation and dual checkpoint blockade activate non-redundant immune mechanisms in cancer. Nature. 2015;520(7547):373-377.

90. Reynders K, Illidge T, Siva S, Chang JY, De Ruysscher D. The abscopal effect of local radiotherapy: using immunotherapy to make a rare event clinically relevant. Cancer Treat Rev. 2015;41(6):503-510.

91. Ang KK, Peters LJ, Weber RS, et al. Postoperative radiotherapy for cutaneous melanoma of the head and neck region. Int J Radiat Oncol Biol Phys. 1994;30(4):795-798.

92. Ang KK, Byers RM, Peters LJ, et al. Regional radiotherapy as adjuvant treatment for head and neck malignant melanoma. Preliminary results. Arch Otolaryngol Head Neck Surg. 1990;116(2):169-172.

93. Agrawal S, Kane JM 3rd, Guadagnolo BA, Kraybill WG, Ballo MT. The benefits of adjuvant radiation therapy after therapeutic lymphadenectomy for clinically advanced, high-risk, lymph node-metastatic melanoma. Cancer. 2009;115(24):5836-5844.

94. Guadagnolo BA, Zagars GK. Adjuvant radiation therapy for highrisk nodal metastases from cutaneous melanoma. Lancet Oncol. 2009;10(4):409-416.

95. Chang DT, Amdur RJ, Morris CG, Mendenhall WM. Adjuvant radiotherapy for cutaneous melanoma: comparing hypofractionation to conventional fractionation. Int J Radiat Oncol Biol Phys. 2006;66(4): 1051-1055.

96. Burmeister BH, Henderson MA, Ainslie J, et al. Adjuvant radiotherapy versus observation alone for patients at risk of lymph-node field relapse after therapeutic lymphadenectomy for melanoma: a randomised trial. Lancet Oncol. 2012;13(6):589-597. 
97. Pinkham MB, Foote MC, Burmeister E, et al. Stage III melanoma in the axilla: patterns of regional recurrence after surgery with and without adjuvant radiation therapy. Int J Radiat Oncol Biol Phys. 2013;86(4):702-708.

98. Mendenhall WM, Shaw C, Amdur RJ, Kirwan J, Morris CG, Werning JW. Surgery and adjuvant radiotherapy for cutaneous melanoma considered high-risk for local-regional recurrence. Am J Otolaryngol. 2013;34(4):320-322.

99. Ahmed S, Kottschade L, Markovic S, Garces Y, Foote R. Adjuvant radiotherapy for melanoma metastatic to axillary and inguinal nodes: regional tumor control and late effects. J Cancer Res Ther. 2014;2(9): $144-152$.

100. Grunewald S, Jank A. New systemic agents in dermatology with respect to fertility, pregnancy, and lactation. J Dtsch Dermatol Ges. 2015;13(4):277-289; quiz 290.

101. Murphy D, Orgel E, Termuhlen A, Shannon S, Warren K, Quinn GP. Why healthcare providers should focus on the fertility of AYA cancer survivors: it's not too late! Front Oncol. 2013;3:248.

102. Coit DG, Thompson JA, Andtbacka R, et al. Melanoma, version 4.2014. J Natl Compr Canc Netw. 2014;12(5):621-629.

103. Ferrari A, Bisogno G, Cecchetto G, et al. Cutaneous melanoma in children and adolescents: the Italian rare tumors in pediatric age project experience. J Pediatr. 2014;164(2):376-382. e371-e372.
104. Parsons HM, Schmidt S, Harlan LC, et al. Young and uninsured: Insurance patterns of recently diagnosed adolescent and young adult cancer survivors in the AYA HOPE study. Cancer. 2014;120(15): 2352-2360.

105. Parsons HM, Harlan LC, Seibel NL, Stevens JL, Keegan TH. Clinical trial participation and time to treatment among adolescents and young adults with cancer: does age at diagnosis or insurance make a difference? J Clin Oncol. 2011;29(30):4045-4053.

106. Zebrack B, Kent EE, Keegan TH, Kato I, Smith AW, Group AHSC. "Cancer sucks," and other ponderings by adolescent and young adult cancer survivors. J Psychosoc Oncol. 2014;32(1):1-15.

107. Barakat LP, Schwartz LA, Reilly A, Deatrick JA, Balis F. A qualitative study of phase III cancer clinical trial enrollment decision-making: perspectives from adolescents, young adults, caregivers, and providers. J Adolesc Young Adult Oncol. 2014;3(1):3-11.

108. GLOBOCAN 2012: Estimated cancer incidence, mortality and prevalence worldwide in 2012. International Agency for Research on Cancer; World Health Organization. Available from: http://globocan. iarc.fr/Pages/age-specific_table_sel.aspx. Accessed August 21, 2015.
Clinical Oncology in Adolescents and Young Adults

\section{Publish your work in this journal}

Clinical Oncology in Adolescents and Young Adults is an international, peer-reviewed, open access journal publishing original research, reports, editorials, reviews and commentaries on all aspects of epidemiology, diagnosis and treatment of cancers in adolescents and young adults. The manuscript management system is completely

\section{Dovepress}

online and includes a very quick and fair peer-review system. Visit http://www.dovepress.com/testimonials.php to read real quotes from published authors. 